\title{
Aspectos do Conhecimento Profissional Sobre Transformações Geométricas Emergentes em uma Atividade Diagnóstica com a Participação de um Grupo de Professores
}

\section{Emerging Aspects of Professional Knowledge about Geometric Transformations in a Diagnostic Activity with the Participation of a Group of Teachers}

\author{
Isabel Campos Barroso; ; Maria Elisa Esteves Lopes Galvão ${ }^{\mathrm{b}}$, Angelica da Fontoura Garcia Silva*b \\ ${ }^{a}$ Colégio Pedro II. SP, Brasil. \\ bUniversidade Anhanguera de São Paulo. SP, Brasil. \\ *E-mail:angelicafontoura@anhanguera.com
}

\begin{abstract}
Resumo
Tem-se como objetivo nesse trabalho trazer parte de uma pesquisa que teve como propósito identificar e analisar o conhecimento profissional docente para o ensino das transformações geométricas na Educação Básica e examinar as contribuições de uma formação voltada para esse conhecimento. Participaram da pesquisa 21 professores do Ensino Fundamental e Médio, que atuam na rede pública do Rio de Janeiro. A investigação seguiu princípios metodológicos do Design Experiment. O aporte teórico sobre o conhecimento docente baseou-se nos estudos de Ball, Thames e Phelps; os trabalhos de Bulf, Grenier e Jahn trouxeram referências importantes sobre as transformações geométricas. Uma atividade diagnóstica para avaliar os conhecimentos sobre os vários tipos de simetria, eixos de reflexão e centros de rotação, precedeu a preparação de um conjunto de atividades que explorou a construção do conhecimento sobre esse conteúdo usando desde materiais manipulativos até um software de Geometria Dinâmica. Traz-se para esse artigo os dados referentes à avaliação diagnóstica e busca-se compreender, dentre os professores participantes, os conhecimentos comum e especializado para o ensino das transformações geométricas emergentes dessa atividade. As respostas à atividade diagnóstica revelaram que os participantes carecem do conhecimento comum e especializado, consequência de uma formação inicial que pouco contemplou esse conteúdo. As atividades e as discussões sobre o tema possibilitaram a ampliação dos conhecimentos profissionais dos professores, especialmente o conhecimento do conteúdo.
\end{abstract}

Palavras-chave: Transformações Geométricas. Simetria. Formação de Professores. Conhecimento Docente. Residência Docente.

\begin{abstract}
The objectives of this work are to bring part of a research that aimed to identify and analyze the teaching professional knowledge for the teaching of geometric transformations in Basic Education and to examine the contributions of some activities focused on this knowledge. Twenty-one elementary and high school teachers from Rio de Janeiro public schools participated in the research. The research followed the methodological principles of Design Experiment. The theoretical support on teaching knowledge was based on the studies of Ball, Thames and Phelps; the works of Bulf, Grenier, and Jahn brought important references about geometric transformations. A diagnostic activity to assess knowledge about the various types of symmetry, reflection axes, and centers of rotation preceded the preparation of a set of activities that explored knowledge building on this content using from manipulative materials to Dynamic Geometry software. This article brings the data related to the diagnostic evaluation and seeks to understand, among the participating teachers, the common and specialized knowledge for teaching the geometric transformations emerging from this activity. The responses to the diagnostic activity revealed that the participants lack common and specialized knowledge, a consequence of an initial formation that had little contemplation of this content. The activities and discussions on the subject allowed the expansion of teachers 'professional knowledge, especially the content knowledge.
\end{abstract}

Keywords: Geometric Transformations. Symmetry. Teacher Training. Teaching Knowledge. Teaching Residency.

\section{Introdução}

As transformações geométricas constituem um campo rico para estabelecer conexões entre os contextos geométrico, algébrico e funcional no ensino da matemática. Em especial, o contexto funcional permite um tratamento diferenciado dos problemas de geometria e fornece ferramentas disponíveis para demonstrações e a resolução de problemas (Grenier, 1988, Thaqi, 2009, Usiskin, 2014). No Brasil, esse conteúdo foi proposto na reforma Francisco Campos de 1931 e no Movimento da Matemática Moderna (Mabuchi, 2000). Permaneceu nas propostas curriculares que se seguiram e mais tarde nos Parâmetros Curriculares Nacionais (PCN) até as recentes da Base Nacional Comum Curricular (BNCC). Entretanto entendemos que as transformações ainda aparecem pouco nos currículos praticados nas escolas e consideramos que precisam ser efetivamente incorporadas aos cursos de formação de professores.

Diversos trabalhos têm se debruçado sobre as transformações geométricas, apresentando razões e contribuições do seu estudo, dentre as quais destacamos: as transformações são aplicações das funções no contexto da Geometria e o tratamento funcional é fundamental em toda a Matemática; as transformações permitem tratar as tarefas geométricas de forma dinâmica (Grenier, 1988, Usiskin, 2014, Thaqi, 2009). Em particular, segundo Bulf (2008), a simetria tem uma dimensão cultural e social, revela as transformações presentes em situações cotidianas, relacionadas à beleza, ordem e perfeição.

Neste estudo consideramos e constatamos as hipóteses 
de que as transformações geométricas não se constituem um conhecimento de conteúdo de amplo domínio no grupo de professores participantes, quer pela formação inicial, quer pela atuação profissional. Nosso propósito, no contexto amplo do trabalho com os professores, foi identificar: quais conhecimentos os professores participantes têm sobre as transformações geométricas no plano e quais as possíveis contribuições de um minicurso sobre transformações geométricas para a ampliação dos conhecimentos dos professores participantes sobre este conteúdo de forma a propiciar que chegue às suas salas de aula.

Apoiamo-nos em duas vertentes teóricas para a construção da nossa pesquisa. Uma direcionada ao objeto matemático do estudo, as transformações geométricas, e outra direcionada à formação do professor e aos conhecimentos docentes. Encontramos nos trabalhos de Grenier (1988), Bulf (2008) e Jahn (1998) aspectos das transformações geométricas enquanto objeto matemático relevantes para o nosso estudo. No que se refere à formação de professores nossa pesquisa se apoia nos resultados de estudos relacionados à formação do professor e ao conhecimento profissional para a docência preconizados por Shulman $(1986,1987)$ e refinados por Ball, Thames, \& Phelps (2008). Daremos destaque, no recorte que apresentamos, aos conhecimentos comum e especializado para o ensino, detalhamentos destinados a compreender os conhecimentos sobre o conteúdo que os professores utilizam em sua prática.

Norteamos o percurso metodológico dessa pesquisa pelo design experiment (Cobb et al., 2003), no intuito de planejar um ambiente educacional e simultaneamente conduzir estudos experimentais por meio da intervenção realizada com um grupo de 19 professores participantes de um programa destinado a professores ingressantes na carreira docente, o Programa de Residência Docente ${ }^{1}$ - PRD- de uma instituição pública do Rio de Janeiro. Para a fase prospectiva do design foram analisados estudos sobre o currículo e os livros didáticos, os dados de entrevistas semiestruturadas realizadas com professores que atuam como formadores no PRD e do questionário diagnóstico respondido pelos professores residentes. A análise dos dados coletados a partir desse instrumento forneceu as bases para o design de um minicurso com atividades utilizando desde material concreto até atividades que devem ser propostas com auxílio de um software de Geometria Dinâmica. Essa formação serviu também para a obtenção de dados sobre como os professores argumentam e lidam com possibilidades para o estudo das transformações geométricas em suas salas de aula.

Para a análise da apresentação das transformações geométricas nos livros didáticos examinamos as 11 coleções recomendadas no Programa Nacional do Livro Didático de 2017. Todas apresentam algum tópico relacionado às transformações geométricas e iniciam o estudo explorando as simetrias. No entanto, o que é privilegiado é a sobreposição de figuras, limitando-se à simetria por reflexão, embora a palavra reflexão nem sempre apareça, gerando a ambiguidade entre o conceito de figura simétrica em relação a uma reta e a simetria de uma figura como a transformação que deixa a figura invariante. Não é claramente estabelecida a relação entre as isometrias e as simetrias correspondentes (Brasil, 2016) e parte-se da reflexão em relação a um eixo vertical. A malha quadriculada é considerada em todos os livros analisados, mas nem todos ampliam a construção sem o uso da malha. De forma geral, as coleções passam a ideia de que a simetria está associada apenas à reflexão, carecendo do reconhecimento da simetria quando há rotação, por exemplo.

A partir da análise dos livros, escolhemos iniciar o estudo empírico partindo de uma atividade diagnóstica visando explorar as propriedades de simetria de conjuntos variados de figuras. Os livros introduzem as transformações explorando as simetrias. Além disso, a simetria é uma noção familiar, traz referências cotidianas, possui uma dimensão cultural (Bulf, 2008, Grenier, 1988), ou seja, o objeto cultural e o conceito matemático entram em interação. Traremos para análise, neste artigo, parte dos dados relacionados à prospecção sobre o conhecimento dos participantes da pesquisa sobre as simetrias realizada na fase diagnóstica que antecedeu a realização do minicurso no Programa de Residência Docente.

\section{Os Olhares Teóricos}

$\mathrm{Na}$ investigação sobre os conhecimentos de professores participantes de um minicurso na residência docente, adotaremos duas vertentes teóricas, a primeira relacionada às transformações geométricas enquanto objeto matemático e a segunda relativa aos conhecimentos docentes para o ensino. Encontramos nas pesquisas de Grenier (1988), Jahn (1998) e Bulf (2008) um olhar interessante sobre as transformações proveniente de três décadas distintas.

Grenier (1988) justifica seu estudo sobre os conhecimentos da simetria ortogonal observando que se trata de uma noção familiar que é privilegiada entre as outras transformações no plano, por gerar, por meio de composições, rotações, translações e reflexões com deslizamento. Grenier (1988) baseou-se em um estudo de Kuchemann \& Hart (1981) que consideraram como variáveis didáticas no estudo das transformações os aspectos relacionados à complexidade da figura (ponto, segmento, reta); à presença ou ausência de malha quadriculada; à posição do eixo de simetria (horizontal, vertical); à distância da figura ao eixo de simetria; e à interseção da figura inicial com o eixo. A pesquisadora verificou uma hierarquia na disponibilidade e aprendizagem dos alunos nas quatro propriedades da simetria ortogonal: posição, ortogonalidade, equidistância e incidência, nessa

1 O Programa de Residência Docente foi criado em 2012 com o objetivo de ser um programa-piloto de parceria entre um colégio público do Rio de Janeiro e a CAPES, para subsidiar a formulação de uma política nacional de formação de professores ingressantes na carreira docente. 
ordem. Observou que quando as variáveis eram a direção oblíqua do eixo e a interseção da figura com a reta de simetria os níveis de dificuldade aumentavam, sendo que neste último nível as dificuldades persistiam mesmo após o ensino. As questões abordadas por Grenier (1988) têm relevância para o contexto desta pesquisa uma vez que procuraremos, dentre os conhecimentos explicitados pelos participantes, as evidências de conhecimentos sobre as propriedades simetria ortogonal, destacadas pela autora, que poderão, de alguma forma, interferir nas demais categorias de conhecimentos necessários ao ensino dessa temática posteriormente. As variáveis didáticas foram consideradas na seleção das atividades que compuseram o minicurso que fez parte desta pesquisa, por entendermos que devem fazer parte do repertório de forma a favorecer o desenvolvimento dos conhecimentos a serem utilizados pelo professor.

Jahn (1998) examinou as dificuldades apresentadas pelos alunos, no tratamento dado as transformações geométricas. Seu objetivo foi analisar condições nas situações didáticas que podem favorecer o aspecto dual global/pontual das transformações e mostrar que a dinâmica propiciada por um software pode contribuir para renovar a dialética global/ pontual e colocar em evidência a relação entre as concepções estática e dinâmica nos problemas de construção de figuras. Jahn (1998) utilizou uma sequência didática na qual observou as dificuldades na passagem entre os níveis de apreensão no trabalho com as transformações geométricas, desenvolvidos por Grenier \& Laborde:

- Nível 1: a transformação é considerada "como uma relação entre duas configurações geométricas ou uma relação entre duas partes da mesma configuração" (neste nível o caráter funcional está ausente);

- Nível 2: a transformação é considerada como uma aplicação pontual do plano sobre ele mesmo (trata-se do objeto funcional);

- Nível 3: a transformação é considerada como uma ferramenta funcional, a fim de colocar em evidência os invariantes ou para fins de resolução de problemas. (Laborde e Grenier, 1987, apud Jahn, 1998, p. 60)

Jahn (1998) realizou um estudo que a levou a acrescentar um quarto nível associado ao estado transfigural e caracterizado pelas estruturas de conjunto:

- Nível 4: a transformação é considerada como um elemento de um grupo (as transformações se compõem e se comparam, elas formam grupos de transformações).

Como resultados, Jahn (1998) considera que seu estudo evidenciou as dificuldades na noção de transformação geométrica como aplicação pontual, ainda no nível 2. Nesse estudo, é-realizado uma ampliação dos estudos feitos até então: enquanto Grenier (1988) realizou atividades que exploram as transformações no primeiro nível de apreensão, a autora procurou trabalhar no segundo nível com o propósito de chegar ao terceiro nível. O percurso desta pesquisa está mais próximo do seguido por Grenier, mas vamos considerar alguns aspectos relacionados aos dois primeiros níveis elencados por Jahn.

Bulf (2008) concentrou sua investigação sobre os efeitos da simetria axial ${ }^{2}$ na aprendizagem das outras isometrias planas e sobre a natureza do trabalho geométrico. Estudou ainda os efeitos da realidade sobre a aprendizagem, tendo como questão central o papel desempenhado por essa transformação na conceituação de outras isometrias, a simetria central e a rotação. Sua questão principal é se a simetria axial funciona como um motor ou um obstáculo para a aprendizagem das outras isometrias. A pesquisadora parte do ideário construtivista de que um conhecimento é construído a partir dos anteriores. Assim como Grenier (1988), Bulf (2008) também teve interesse em investigar como o estudante pensa e quais fatores podem influir de forma positiva ou negativa na identificação mais rápida de uma simetria axial e como interpreta o movimento, no caso dessa transformação. Um dos aspectos importantes observados por Bulf (2008) é o fato de que se sente a necessidade de um ajuste corporal, quando o eixo de simetria é inclinado, virando a cabeça para coincidir os eixos de visão e da simetria. Aborda os aspectos relacionados ao seu uso cotidiano, que considera culturais, como, por exemplo, a prática dos artesãos, e analisa se essas noções cotidianas funcionam como alavanca ou não para compreensão da transformação. A autora conclui que a simetria axial pode desempenhar um papel organizador nas atividades do estudante ou de um artesão, mas pode causar entraves em alguns alunos devido à proximidade com os esquemas de simetria central.

Em nossa pesquisa consideraremos elementos relacionados aos percursos utilizados por Grenier (1988), Jahn (1998) e Bulf (2008) que direcionaram suas investigações para as transformações do ponto de vista "pontual ou local", abordagens adotadas para as transformações nos livros didáticos e prescritas nas orientações curriculares brasileiras. Enquanto as pesquisadoras observaram a aprendizagem dos alunos frente às variáveis didáticas, buscamos confrontar os resultados observados por elas com grupo de professores em início de carreira aqui investigados.

Estudos sobre os conhecimentos do professor têm marcado as pesquisas na área de formação de professores, inicial ou continuada. Os pesquisadores Ball, Thames e Phelps (2008) tomam como referência as ideias de Shulman (1986, 1987), direcionando o foco de suas investigações aos conhecimentos do professor para o ensino de Matemática. Ball e seus colaboradores desenvolvem a noção de conhecimento matemático para o ensino ${ }^{3}$, influenciados pela noção de conhecimento pedagógico de conteúdo e tomando como base a observação e análise de aspectos da prática de sala de aula para complementar essas noções. 
Por 'conhecimento matemático para o ensino', entendemos o conhecimento matemático necessário para realizar o trabalho de ensino da matemática. É importante notar aqui que nossa definição começa com ensino, não com os professores. Preocupa-nos as tarefas envolvidas no ensino e as exigências matemáticas dessas $\operatorname{tarefas}^{4}$ (Ball, Thames, \& Phelps, 2007, p. 395).

Seus estudos conduzem a um "refinamento para o popular conceito de conhecimento pedagógico do conteúdo e ao conceito mais amplo de conhecimento de conteúdo para o ensino" (Ball, Thames, \& Phelps, 2008, p. 390). O conhecimento de matemática para o ensino, segundo as ideias de Ball e seus colaboradores (2008) envolve saber mais do que se ensina. "um professor precisa saber mais matemática e de forma diferente - não menos" ${ }^{6}$ " (Ball, Thames, \& Phelps, 2008. p. 396) e propõem um modelo a partir das análises desenvolvidas sobre as demandas matemáticas para o ensino. Esse modelo fundamenta-se em subdivisões de duas das categorias propostas por Shulman $(1986,1987)$ a saber, o conhecimento de conteúdo e o conhecimento pedagógico de conteúdo, ampliando as categorias de conhecimentos, subdividindo cada uma dessas categorias em três, que devem levam em conta, segundo os autores, a natureza, o papel e a importância dos diferentes tipos de conhecimento matemático para o ensino.

O conhecimento de conteúdo, segundo Ball, Thames \& Phelps (2008) subdivide-se em: conhecimento comum do conteúdo (CCK), conhecimento especializado do conteúdo ${ }^{7}$ (SCK), e conhecimento de horizonte do conteúdo (HCK); o conhecimento pedagógico de conteúdo divide-se em: conhecimento do conteúdo e do ensino (KCT); conhecimento do conteúdo e dos alunos (KCS) e conhecimento do conteúdo e do currículo (KCC).

Neste estudo analisaremos o Conhecimento Comum do Conteúdo e o Conhecimento Especializado do Conteúdo emergentes dos dados coletados. Além disso, ao observarmos a complementaridade entre essas categorias também optamos por agrupá-las e denominá-los conhecimento do conteúdo comum/especializado. Assim, por fazer parte do escopo deste estudo as descreveremos a seguir.

O conhecimento matemático é comum a um número diversificado de profissões, que se utilizam da matemática na sua atuação profissional, dessa forma o conhecimento comum de conteúdo é um saber para si próprio, um conhecimento sobre a matemática que é utilizado por todos os que se valem do conhecimento matemático para saber fazer. É um conhecimento que não é exclusivo do professor, qualquer pessoa com formação em matemática deve ter. Compreende por exemplo, segundo os autores, o conhecimento para fazer um cálculo, resolver corretamente um problema rotineiro. Em se tratando das transformações geométricas, compreende a habilidade de reconhecê-las nas múltiplas representações geométricas que nos cercam e identificar seus principais elementos, como por exemplo, eixos de reflexão ou simetria, centros de rotação, direções de translação.

Por outro lado, deseja-se e faz-se necessário que os professores possuam um conhecimento amplo, rico e profundo sobre o conteúdo de modo a levar a cabo o ensino de matemática, compreender e ter condições de discutir distintas alternativas para a resolução de um problema. O conhecimento especializado do conteúdo é um conhecimento específico do professor e que não é necessário em outras situações que não seja o ensino. Envolve saber responder os porquês, as diferentes representações para um mesmo conteúdo, navegar de forma eficaz entre elas e entender os motivos dos tipos de erros dos alunos. É um conhecimento além do saber fazer, saber ensinar a fazer, envolve todo saber que vá além de somente saber dizer se está correto ou incorreto. No caso das transformações, podemos considerar, entre outros aspectos, por exemplo, seu estudo de ponto de vista funcional, a compreensão das propriedades de composição, a relação com a congruência, os níveis de compreensão explicitados por Jahn (1998).

As pesquisas voltadas para a compreensão dos conhecimentos para o ensino destacam a importância da prática na produção dos saberes docentes e a reflexão sobre a formação dos professores. Nesse sentido, reconhecemos como principal contribuição desses estudos, a percepção de um conhecimento especializado para o ensino da matemática. Além disso, a construção desse conhecimento não se esgota na formação inicial, sendo um processo permanente que visa o desenvolvimento do olhar de maneira profissional.

\section{Material e Métodos}

Os 21 professores que fizeram parte deste estudo são professores da rede pública do Rio de Janeiro, com seis anos de magistério, em média, divididos em dois grupos de trabalho que denominaremos G1 e G2 com, respectivamente, 10 e 9 participantes, em razão da disponibilidade de horário dos participantes. Apenas dois dentre eles consideram que seus estudos em Geometria tenham sido satisfatórios, tanto na educação básica quanto no ensino superior. Quanto às transformações geométricas, 14 informaram haver visto o assunto, em disciplinas como diversas, sem citar disciplinas de Geometria, outros disseram ter estudado por conta própria, em livros, independente das disciplinas cursadas. Cinco dentre os professores relataram não ensinar Geometria e somente

4 No original "By "mathematical knowledge for teaching," we mean the mathematical knowledge needed to carry out the work of teaching mathematics. Important to note here is that our definition begins with teaching, not teachers. It is concerned with the tasks involved in teaching and the mathematical demands of these tasks".

5 No original "refinements to the popular concept of pedagogical content knowledge and to the broader concept of content knowledge for teaching" 6 No original "In short, a teacher needs to know more, and different, mathematics - not less".

7 Specialized content knowledge 
duas professoras disseram já ter ensinado transformações geométricas.

O minicurso foi planejado para cinco encontros para os dois grupos de professores. O primeiro encontro foi destinado à avaliação diagnóstica, os três encontros seguintes foram dedicados ao estudo da reflexão, da translação e da rotação e à uma discussão sobre a apresentação das transformações geométricas nos livros didáticos. Ao longo do minicurso os participantes prepararam atividades para levar às suas salas de aula e no encontro final aconteceu a apresentação dos resultados das atividades preparadas pelos residentes.

Traremos para análise dados relativos a parte das atividades propostas no diagnóstico inicial; buscamos atividades que envolvessem as variáveis didáticas sobre as transformações geométricas (Bulf, 2008, Grenier, 1988): complexidade da figura, posição do eixo de simetria, distância ao eixo, interseção da figura com o eixo; e que contemplassem os níveis 1 e 2 de apreensão no trabalho com as transformações geométricas (Grenier, 1988, Jahn, 1998), ou seja, a transformação como uma relação entre duas configurações geométricas ou entre duas partes de uma figura e a transformação como uma aplicação do plano sobre ele mesmo. Quanto aos conhecimentos para o ensino (Ball et al., 2008, Shulman, 1988), direcionamos nossa atenção especialmente aos conhecimentos comum e especializado sobre as transformações geométricas, no momento do diagnóstico que trazemos para análise.

Para a primeira atividade do diagnóstico, selecionamos 30 logotipos, incluindo exemplos de simetria por reflexão em relação a eixos vertical, horizontal ou inclinados, simetria por rotação e exemplos sem simetria (Figura 1).Observe os vários logotipos a seguir, indique quais possuem alguma simetria e identifique a simetria e seus elementos principais.

Figura 1 - Logotipos

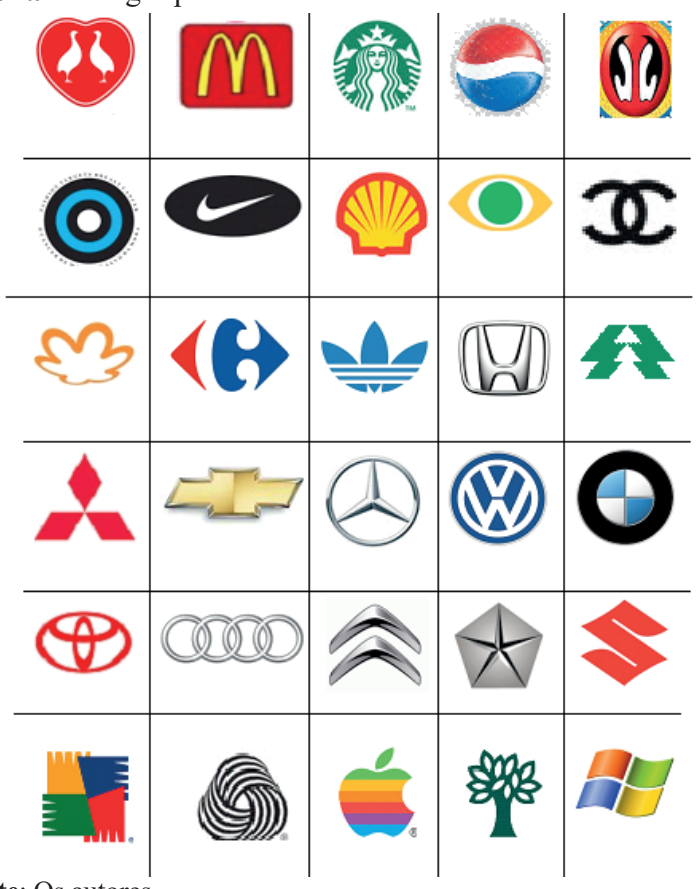

Os professores foram solicitados a sinalizar os logos que possuem simetria, indicar qual a isometria identificada, destacando os seus elementos. O objetivo foi verificar: se os professores distinguem quais possuem simetria; se reconhecem as isometrias que mantém a figura invariante; e reconhecem elementos como eixos de simetria, centro e ângulo de rotação. Consideramos que esses aspectos nos levariam à identificação de elementos do conhecimento dos professores relacionados ao à base de conhecimento comum/especializado, conforme Ball, Thames \& Phelps (2008). A análise da atividade foi realizada agrupando os logotipos por tipo de simetria, de forma a evidenciar os conhecimentos relacionados a cada um dos tipos de simetria.

\section{Resultados e Discussão}

O primeiro bloco de logotipos selecionado para a análise das indicações e identificações contidas nos registros dos protocolos dos residentes foi o bloco formado por logotipos nos quais se identifica uma simetria de reflexão, ou simetria axial, cujo eixo de reflexão é vertical, reproduzidos na Figura 2.

Figura 2 - Logotipos com eixo de reflexão vertical

\begin{tabular}{|l|l|l|}
\hline & & \\
\hline
\end{tabular}

Fonte: Os autores.

Entendemos que por ser um instrumento destinado ao diagnóstico, os aspectos analisados dizem respeito ao conhecimento comum/especializado para o ensino das transformações geométricas, ou seja, tais como: identificação da simetria; reconhecimento da isometria envolvida, reflexão em relação a uma reta e rotação, incluindo a simetria central; identificação dos parâmetros envolvidos: eixos de simetria, considerar a influência da variável didática da posição dos eixos, no caso da reflexão; e centro e ângulo de rotação, verificar quais as variáveis que podem interferir neste caso. Consideramos que esse conhecimento envolve além do conhecimento do saber fazer - reconhecimento -, mas também o saber ensinar a fazer, pois envolve conhecimentos de propriedades das transformações que permitirão ao 
professor discutir matematicamente sobre a temática que está ensinando.

Procedemos às análises das resoluções dos 19 participantes desse diagnóstico, por grupos de logotipos, complementada por extratos das discussões posteriormente realizadas. no Quadro 1 estão as respostas dadas ao subgrupo dos logotipos que possuem simetria por reflexão em relação a um eixo vertical.

Quadro 1 - Respostas aos logotipos com reflexão em relação a um eixo vertical

\begin{tabular}{|c|c|c|c|c|c|c|c|c|c|}
\hline & Logo $\backslash$ Resposta & \multicolumn{2}{|c|}{ Identifica Simetria } & \multicolumn{2}{|c|}{ Reflexão/Axial } & \multicolumn{2}{|c|}{ Eixo Vertical } & \multicolumn{2}{|c|}{ Outras } \\
\hline & Grupos & G1 & G2 & G1 & G2 & G1 & G2 & G1 & G2 \\
\hline A & & 3 & 2 & 5 & 4 & 10 & 8 & Rotação 1 & \\
\hline B & & 3 & 2 & 6 & 4 & 10 & 8 & & \\
\hline $\mathrm{C}$ & & 3 & 2 & 6 & 4 & 10 & 8 & & \\
\hline $\mathrm{D}$ & & 3 & 2 & 6 & 4 & 10 & 8 & & \\
\hline $\mathrm{E}$ & & 4 & 1 & 4 & 4 & 10 & 7 & $\begin{array}{c}\text { Não sei } \\
1\end{array}$ & $\begin{array}{c}\text { Não tem } \\
1\end{array}$ \\
\hline $\mathrm{F}$ & & 4 & 2 & 5 & 4 & 11 & 8 & & \\
\hline G & & 4 & 1 & 4 & 4 & 10 & 8 & $\begin{array}{c}\text { Em branco } \\
1\end{array}$ & $\begin{array}{c}\text { Não } \\
1\end{array}$ \\
\hline $\mathrm{H}$ & & 4 & 2 & 5 & 3 & 10 & 8 & & \\
\hline I & & 4 & 2 & 5 & 2 & 11 & 8 & & $\begin{array}{c}\text { Translação } \\
1\end{array}$ \\
\hline $\mathrm{J}$ & & 4 & 2 & 4 & 1 & 10 & 8 & $\begin{array}{c}\text { Não sei } \\
1\end{array}$ & $\begin{array}{c}\text { Não sei } \\
\text { translação } \\
2\end{array}$ \\
\hline $\mathrm{K}$ & CITROËn & 4 & 2 & 4 & 3 & 9 & 8 & $\begin{array}{c}\text { Não sei } \\
1\end{array}$ & \\
\hline
\end{tabular}

Fonte: Os autores.

Em cada um dos grupos, somente um dos participantes não identificou o eixo vertical; o eixo foi corretamente identificado em quase todos os logos, com exceção do último (K). Com relação ao reconhecimento da isometria, alguns residentes respondem reflexão, outros, axial, e um, reflexão axial. Alguns residentes não identificaram a isometria envolvida, apenas marcaram $\mathrm{X}$ ou escreveram sim para indicar que há simetria, no G1, e escreveram simetria, no G2. Outros escreveram uma observação no fim da folha "Não lembro os nomes das simetrias", mas desenharam o eixo vertical. Esta foi a principal dificuldade detectada, a necessidade da identificação da transformação para definir a simetria, ou seja, essa identificação não está presente no conhecimento comum sobre transformações. Observamos, já nessa fase inicial de análise, que os conhecimentos básicos sobre simetria precisam ser explorados no minicurso, ou seja, que o conhecimento do conteúdo comum/especializado precisa ser enriquecido pois, inclusive no caso com mais acertos, quando há reflexão em 
relação a um eixo vertical, identificamos elementos que não são de domínio de todos os componentes do grupo.

No Quadro 2, estão as respostas dadas no logo em que há simetria apenas por reflexão em relação a um eixo horizontal.
Com relação à identificação do eixo, o resultado foi próximo do anterior, apenas dois não o identificaram: um deixou em branco e outro não desenhou o eixo, respondendo apenas "rotação".

Quadro 2 - Respostas explicitadas ao logotipo com reflexão em relação a um eixo horizontal

\begin{tabular}{|c|c|c|c|c|c|c|c|c|c|c|}
\hline Logo/Resposta & \multicolumn{2}{|c|}{$\begin{array}{c}\text { X ou sim/ } \\
\text { simetria }\end{array}$} & \multicolumn{2}{|c|}{ Reflexão / Axial } & \multicolumn{2}{|c|}{ Eixo horizontal } & \multicolumn{2}{|c|}{ Rotação } & \multicolumn{2}{c|}{ Em branco } \\
\hline Grupos & G1 & G2 & G1 & G2 & G1 & G2 & G1 & G2 & G1 & G2 \\
\hline Carrefour & 3 & 2 & 4 & 3 & 10 & 7 & 1 & & & 1 \\
\hline
\end{tabular}

Fonte: Dados da pesquisa.

Já quanto ao reconhecimento da isometria, o resultado foi inferior ao anterior, mantendo-se o número de cinco professores que apenas reconheceram haver simetria. Portanto, a variável posição do eixo horizontal não interferiu na identificação da simetria ou do eixo, mas houve uma redução na identificação da isometria, em razão da mudança de posição do eixo. Esse fato corrobora pesquisas como a de Grenier (1988) no que se refere à maior facilidade na identificação da simetria quando o eixo de reflexão é vertical.

No Quadro 3, apresentamos as respostas dos residentes aos logotipos que possuem dois eixos de simetria, um horizontal e um vertical.

Quadro 3 - Respostas aos logotipos com eixos de simetria horizontal e vertical

\begin{tabular}{|c|c|c|c|c|c|c|c|c|c|c|c|c|}
\hline \multirow{3}{*}{$\begin{array}{l}\text { Logo\Resposta } \\
\text { Grupos }\end{array}$} & \multirow{2}{*}{\multicolumn{2}{|c|}{$\begin{array}{l}\mathrm{X} \text { ou sim / } \\
\text { simetria }\end{array}$}} & \multirow{2}{*}{\multicolumn{2}{|c|}{$\begin{array}{c}\text { Reflexão ou } \\
\text { Axial } \\
\text { Horizontal }\end{array}$}} & \multicolumn{6}{|c|}{ Desenhou os eixos } & \multirow{2}{*}{\multicolumn{2}{|c|}{ Outras }} \\
\hline & & & & & \multicolumn{2}{|c|}{ vertical } & \multicolumn{2}{|c|}{$\begin{array}{c}\text { horizontal e } \\
\text { vertical }\end{array}$} & \multirow[b]{2}{*}{ G1 } & \multirow[b]{2}{*}{ G2 } & & \\
\hline & G1 & G2 & G1 & G2 & G1 & G2 & G1 & G2 & & & G1 & G2 \\
\hline & \multirow[b]{2}{*}{4} & \multirow[b]{2}{*}{2} & \multirow{2}{*}{5} & \multirow[b]{2}{*}{3} & \multirow{2}{*}{1} & \multirow[b]{2}{*}{5} & \multirow[b]{2}{*}{4} & & \multirow[b]{2}{*}{5} & \multirow[b]{2}{*}{3} & \multicolumn{2}{|c|}{ Rotação } \\
\hline & & & & & & & & & & & & 1 \\
\hline & \multirow[b]{2}{*}{5} & \multirow[b]{2}{*}{2} & \multirow[b]{2}{*}{4} & \multirow[b]{2}{*}{3} & \multirow[b]{2}{*}{1} & \multirow[b]{2}{*}{1} & \multirow[b]{2}{*}{4} & \multirow[b]{2}{*}{2} & \multirow[b]{2}{*}{5} & \multirow[b]{2}{*}{5} & \multicolumn{2}{|c|}{ Rotação } \\
\hline & & & & & & & & & & & 1 & 1 \\
\hline & \multirow[b]{2}{*}{5} & \multirow[b]{2}{*}{2} & \multirow[b]{2}{*}{4} & \multirow[b]{2}{*}{3} & \multirow[b]{2}{*}{2} & & \multirow[b]{2}{*}{2} & \multirow[b]{2}{*}{3} & \multirow[b]{2}{*}{5} & & & \\
\hline & & & & & & & & & & 5 & 1 & \\
\hline
\end{tabular}

Fonte: Dados da pesquisa.

As respostas deste grupo foram similares quanto à identificação dos eixos, mas variou quanto aos eixos indicados. Embora a maioria (10 residentes nos dois últimos, e 8 no primeiro logo) tenha identificado os eixos horizontal e vertical, dentre os demais, exceto no primeiro logotipo deste grupo, houve um certo predomínio do eixo vertical em relação ao horizontal. Chama a atenção o fato de que dois professores terem dito que havia rotação em alguns desses logos. De fato, há uma rotação de $180^{\circ}$ nestes logotipos, mas não é possível afirmar se de fato a identificaram ou se foi uma resposta ao acaso.

Separamos a seguir os logotipos que possuem outras simetrias, ou seja, mais de dois eixos de simetria (Figura 3), figuras com simetria por reflexão central, ou com eixos distintos do vertical e horizontal.
Figura 3 - Outras simetrias

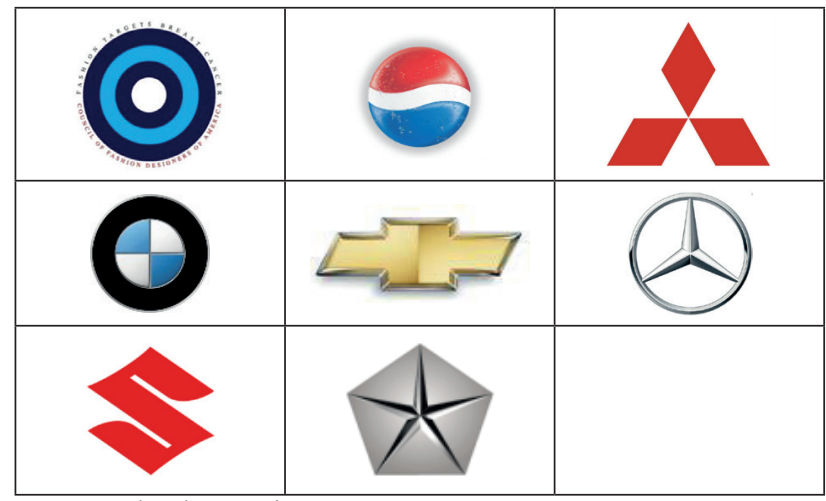

Fonte: Dados da pesquisa.

No Quadro 4, temos as respostas dadas para o primeiro subgrupo desse conjunto de logos. 
Quadro 4 - Respostas aos logos com dois ou infinitos eixos de simetria

\begin{tabular}{|c|c|c|c|c|c|c|c|c|c|c|c|c|c|}
\hline \multirow{3}{*}{$\begin{array}{c}\text { Respostas } \\
\text { / Logos }\end{array}$} & \multirow{2}{*}{\multicolumn{2}{|c|}{$\begin{array}{l}\mathrm{X} / \text { sim } \\
\text { simetria }\end{array}$}} & \multirow{2}{*}{\multicolumn{2}{|c|}{$\begin{array}{c}\text { Reflexão } \\
\text { Axial }\end{array}$}} & \multirow{2}{*}{\multicolumn{2}{|c|}{$\begin{array}{c}\text { Rotação } \\
\text { radial } \\
1 \text { Eixo } \\
\text { vertical }\end{array}$}} & \multirow{2}{*}{$\begin{array}{c}\text { Em } \\
\text { branco } \\
1 \text { eixo } \\
\text { horizontal }\end{array}$} & \multicolumn{6}{|c|}{ Identificou eixos de simetria } \\
\hline & & & & & & & & 3 eixos & $\begin{array}{c}4 \\
\text { Eixos }\end{array}$ & \multirow[b]{2}{*}{1} & \multirow[b]{2}{*}{1} & \multirow[b]{2}{*}{1} & \multirow[b]{2}{*}{1} \\
\hline & 3 & 2 & 4 & 2 & 1 & 2 & 3 & 1 & & & & & \\
\hline & \multicolumn{2}{|c|}{2} & \multicolumn{2}{|c|}{2} & \multicolumn{2}{|c|}{3} & & & 3 & \multicolumn{2}{|c|}{1} & \multicolumn{2}{|c|}{2} \\
\hline
\end{tabular}

Fonte: Dados da pesquisa.

Durante a discussão da atividade alguns residentes perceberam que, de fato, para o primeiro logo havia infinitos eixos de simetria. Um dos participantes perguntou se não seria o caso de uma "simetria radial", resposta que já havia aparecido anteriormente. A rotação foi identificada, neste caso, por apenas $17 \%$ dos residentes. No momento da discussão, sinalizamos que nestes logos existe mais de um eixo de simetria e possuem simetria radial e simetria por rotação, sendo o centro de rotação a interseção de dois dos eixos de simetria.

No segundo logo deste quadro, todos os participantes do G2 identificaram pelo menos um eixo de simetria, mas mesmo com a ajuda do atributo da cor, que poderia levá-los a considerar os eixos inclinados, apenas 3 residentes o fizeram. Isso corrobora o que vimos na literatura. Bulf (2008, p.15), em sua tese, pondera que "o eixo vertical mediano da simetria bilateral aparece como um forte estímulo inicial e vai até provocar "preferências" na percepção que inibiriam outros fenômenos potenciais" ". Ela recorre a estudos da psicologia que apontam a ideia de uma rotação mental ou até mesmo um movimento da cabeça que usualmente fazemos para recuperar o eixo vertical para justificar essa "preferência". Alguns ficaram relutantes em aceitar a ideia de rotação. Algumas manifestações explicitaram dúvidas pertinentes, dentre as quais destacamos: "mas aí tem que girar no plano? Não pode girar no espaço? Então essa simetria que eu tô pensando não existe? Que é como se eu tivesse a axial ali e eu rodasse no espaço, o pedaço direito..." que foi complementada, para que a pesquisadora entendesse a dúvida: "Eu acho que você está falando de quando gira um retângulo pra virar um cilindro", "um sólido de revolução." A discussão foi oportuna, propiciou esclarecimentos sobre as diferenças entre as ações, enquanto "movimento", das transformações e, em particular, as simetrias no plano e no espaço.

As questões mostram que o "movimento" implícito na reflexão não estava claro, e nos fizeram compreender uma identificação inicial, contida no primeiro quadro de respostas, da reflexão axial no plano como rotação, nos dados do primeiro subgrupo de logos. A participante identificou a reflexão axial no plano como uma rotação axial no espaço. Uma interferência semelhante estava ocorrendo na visualização do movimento da rotação, também identificada como a rotação em torno de um eixo perpendicular ao plano da figura e passando pelo seu centro (Figura 4). Nesse momento, a pesquisadora constatou que era importante organizar os conceitos e recuperar a definição da reflexão axial e da rotação no plano do ponto de vista pontual.

As informações dos protocolos para os logos que possuem apenas a simetria central ou rotação de $180^{\circ}$ estão apresentadas no quadro 5. Este grupo foi o que suscitou mais dúvidas e apresentou grande dificuldade para os residentes.

Quadro 5 - Respostas aos logotipos com rotação de $180^{\circ}$

\begin{tabular}{|c|c|c|c|c|c|c|c|c|c|c|c|c|c|c|}
\hline \multirow{3}{*}{$\frac{\text { Logo } \backslash \text { Resposta }}{\text { Grupo }}$} & \multirow{2}{*}{\multicolumn{2}{|c|}{$\begin{array}{c}\mathrm{X} / \operatorname{sim} \\
\text { Simetria }\end{array}$}} & \multirow{2}{*}{\multicolumn{2}{|c|}{ Rotação }} & \multirow{2}{*}{\multicolumn{2}{|c|}{ Em branco }} & \multirow{2}{*}{\multicolumn{2}{|c|}{ Não }} & \multicolumn{4}{|c|}{ Desenhou eixos } & \multirow{2}{*}{\multicolumn{2}{|c|}{$\begin{array}{c}\text { Horizontal } \\
\text { vertical }\end{array}$}} \\
\hline & & & & & & & & & \multicolumn{2}{|c|}{ Vertical } & \multicolumn{2}{|c|}{ Horizontal } & & \\
\hline & G1 & G2 & G1 & G2 & G1 & G2 & \multicolumn{2}{|c|}{ G1 } & G1 & G2 & G1 & G2 & G1 & G2 \\
\hline & & & 3 & 3 & 4 & 4 & \multicolumn{2}{|c|}{4} & & 1 & & 5 & 1 & \\
\hline$\square \square$ & 3 & 1 & 3 & 3 & 4 & & \multicolumn{2}{|c|}{1} & 4 & 6 & & & 1 & 2 \\
\hline \multirow{4}{*}{ Logo \Resposta } & \multirow{2}{*}{\multicolumn{2}{|c|}{$\begin{array}{c}\mathrm{X} / \operatorname{sim} \\
\text { Simetria }\end{array}$}} & \multirow{2}{*}{\multicolumn{2}{|c|}{ Rotação }} & \multirow{2}{*}{\multicolumn{2}{|c|}{ Em branco }} & \multirow{2}{*}{\multicolumn{2}{|c|}{$\begin{array}{c}\text { Reflexão } \\
\text { Axial }\end{array}$}} & Tranclacão & \multicolumn{5}{|c|}{ Desenhou eixos } \\
\hline & & & & & & & & & nstaçao & Vertical & $\mathrm{Hol}$ & ntal & Inc & ado \\
\hline & G1 & $\mathrm{G} 2$ & G1 & $\mathrm{G} 2$ & & & G1 & $\mathrm{G} 2$ & G1 & G1 & G1 & G2 & G1 & $\mathrm{G} 2$ \\
\hline & 2 & 1 & 2 & 3 & & & 1 & 3 & 1 & 3 & 1 & 4 & 2 & 4 \\
\hline
\end{tabular}

Fonte: Dados da pesquisa.

8 No original : «L'axe vertical médian de la symétrie bilatérale apparaît comme un stimulus très fort et va jusqu'à provoquer des « préférences » dans la perception qui inhiberaient d'autres phénomènes potentiels » 
Constatamos que para esses logotipos, em particular, eles precisaram fazer consultas entre si. Observaram que no primeiro logo, a simetria pode ser identificada uma vez que se desconsidere o colorido. É evidente que alguns dos residentes têm uma lembrança de que há outros casos de simetria, como a rotação, mas os conhecimentos sobre a rotação são vagos para eles. Isto fica claro no último logotipo deste grupo, no qual a maioria desenhou um eixo de simetria, horizontal, vertical ou mesmo inclinado. Faltam conhecimentos sobre o conteúdo, tanto do ponto de vista comum, necessário para realizar tarefas sobre o assunto como do ponto de vista especializado, que permitam distinguir as transformações no plano e as espaciais, cuja utilização fica explícita na reprodução do protocolo na Figura 4.
Figura 4 - Respostas de uma participante aos logotipos com rotação de $180^{\circ}$.

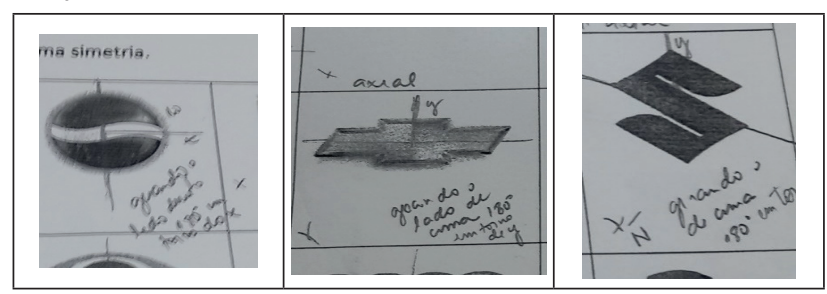

Fonte: Dados da pesquisa.

Os logotipos do quadro 5 foram marcados pela maioria dos residentes como tendo apenas simetria axial, mas na discussão com o grupo surgiram comentários sobre a presença de rotação.

Quadro 6 - Logotipos com simetria por reflexão e rotação

\begin{tabular}{|c|c|c|c|c|c|c|c|c|c|c|c|c|c|c|c|c|}
\hline \multirow{3}{*}{$\begin{array}{c}\begin{array}{c}\text { Logo } \\
\text { resposta }\end{array} \\
\text { Grupos }\end{array}$} & \multirow{2}{*}{\multicolumn{2}{|c|}{$\begin{array}{c}\mathrm{X} / \mathrm{sim} \\
\text { simetria }\end{array}$}} & \multirow{2}{*}{\multicolumn{2}{|c|}{ Reflexão }} & \multirow{2}{*}{\multicolumn{2}{|c|}{ Rotação }} & \multirow{2}{*}{\multicolumn{2}{|c|}{$\begin{array}{c}\text { Em branco } \\
\text { vertical }\end{array}$}} & \multirow{3}{*}{$\begin{array}{l}\text { Não } \\
\text { sei }\end{array}$} & \multirow{2}{*}{\multicolumn{2}{|c|}{ Não }} & \multicolumn{4}{|c|}{ Desenhou eixos } & \multirow{3}{*}{ Ângulo } \\
\hline & & & & & & & & & & & & \multicolumn{2}{|c|}{ vertical } & \multicolumn{2}{|c|}{$3 / 4 / 5$} & \\
\hline & G1 & G2 & G1 & $\mathrm{G} 2$ & G1 & $\mathrm{G} 2$ & G1 & G2 & & G1 & $\mathrm{G} 2$ & G1 & G2 & G1 & G2 & \\
\hline & 4 & 2 & 5 & 1 & 2 & 1 & & 1 & & & & 9 & 5 & 2 & 2 & 1 \\
\hline & 6 & 2 & 4 & 2 & 1 & 1 & 2 & 1 & & & & 1 & 1 & 2 & 3 & 1 \\
\hline & 5 & 2 & 4 & 3 & 2 & 1 & & & & & & & & 1 & 1 & 1 \\
\hline
\end{tabular}

Fonte: Dados da pesquisa.

De fato, estes logotipos, além da simetria axial, com 3 eixos nos dois primeiros e cinco no último, também possuem simetria rotacional. Apenas uma participante indicou o ângulo de rotação, $120^{\circ}$, nos dois primeiros e $72^{\circ}$ no terceiro.

Observamos que a rotação não faz parte do repertório de conhecimento dos residentes sobre as transformações geométricas. Houve dificuldade em identificar as rotações e, na análise de alguns livros didáticos, percebemos que esse tipo de transformação é frequentemente omitido. Estes resultados são compatíveis com a literatura:

Não só a simetria é mais saliente sobre um eixo vertical, mas parece que essa é a única orientação em que uma figura simétrica será percebida espontaneamente como simétrica. Realizamos algumas experiências preliminares em que figuras, simétricas sobre apenas um eixo, são mostradas em várias orientações [...] Os indivíduos são convidados a informar se cada figura parece ou não simétrica. Para a maioria, somente quando o eixo é vertical a figura é percebida como simétrica (Grenier, 1988, p. 16, tradução nossa). ${ }^{9}$

No G2 surgiu uma pergunta muito interessante: "sempre que existe simetria por rotação também vai ter reflexão?”. O participant ${ }^{e}$ constatou que em todos os logos incluídos no Quadro 6, havia rotação e também reflexão. Essa questão foi muito pertinente e repassada para o grupo refletir. Os residentes não conseguiram visualizar uma figura que retratasse essa situação, mas não tiveram certeza para responder negativamente à pergunta. A pesquisadora então desenhou no quadro branco a figura de um cata-vento com dois segmentos perpendiculares e um triângulo em cada ponta deles, como a figura de uma das questões da OBMEP, utilizada no diagnóstico (Figura 5), para orientar a discussão.

9 "Not only is symmetry more salient about a vertical axis, but it would seem that this is the only orientation in which a symmetrical figure will spontaneously be perceived as symmetrical. We have performed some preliminary experiments in which figures, symmetrical about one axis only, are shown in various orientations. Each is presented in only one orientation and it is assumed to be upright in space. The subjects are asked to report whether or not each figure appears symmetrical. For the most part only when the axis is vertical is a figure perceived as symmetrical". (Rock \& Leaman, 1963, apud Grenier, 1988). 
Figura 5 - Catavento

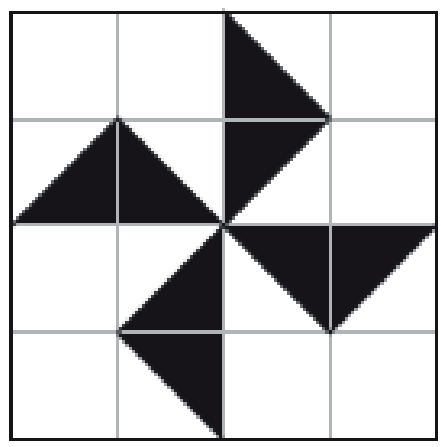

Fonte: Banco de provas OBMEP

Os demais logotipos que completam a atividade dos logos são casos sem simetria ou que "quase tem simetria". Alguns foram prontamente identificados desta forma, mas outros geraram muita discussão durante a realização da atividade e na discussão em grupo depois da atividade entregue, o que pode ser uma justificativa para a multiplicidade de possibilidades de respostas constatadas nos protocolos, exibidas nos Quadros 6 e 7.
Quadro 7 - Logotipos sem simetria

\begin{tabular}{|c|c|c|c|c|c|c|}
\hline Logo\Resposta & \multicolumn{2}{|c|}{ Não } & \multicolumn{2}{c|}{$\begin{array}{c}\text { Em } \\
\text { branco }\end{array}$} & Não sei & $\begin{array}{c}\text { Eixo } \\
\text { vertical }\end{array}$ \\
\hline Grupo & G1 & G2 & G1 & G2 & G1 & G2 \\
\hline & 4 & 2 & 6 & 6 & 1 & \\
\hline & 4 & 1 & 6 & 5 & 1 & 2 \\
\hline 000 & 4 & 2 & 6 & 6 & 1 & \\
\hline
\end{tabular}

Fonte: Os autores.

O logotipo do quadro 7 foi considerado sem simetria por alguns dos professores, mas duas residentes desenharam um eixo um pouco inclinado. Os argumentos usados foram de que a figura representa uma perspectiva de uma figura no espaço que possui simetria.

Quadro 8 - Logotipo com aspecto tridimensional

\begin{tabular}{|c|c|c|c|c|c|c|c|c|c|}
\hline Logo\Resposta & \multicolumn{2}{|c|}{ Não } & \multicolumn{2}{c|}{ Em branco } & Axial & Simetria & \multicolumn{2}{|c|}{1 eixo } & eixos \\
\hline Grupo & $\mathrm{G} 1$ & $\mathrm{G} 2$ & $\mathrm{G} 1$ & $\mathrm{G} 2$ & $\mathrm{G} 1$ & $\mathrm{G} 2$ & $\mathrm{G} 1$ & $\mathrm{G} 2$ \\
\hline
\end{tabular}

Fonte: Os autores.

O último grupo de logotipos suscitou muitas discussões e as respostas variaram (Quadro 8). Pouco mais da metade dos residentes considera que não há simetria, $37 \%$ consideram que há rotação, no segundo logo, e 28\% no primeiro e terceiro, e apenas 2 residentes responderam que há simetria ou axial, nos dois primeiros e 1 no último. Tanto esses como alguns que responderam rotação também desenharam eixos. Foram consideradas as questões das cores e da tridimensionalidade das figuras, se teriam ou não influência para a simetria.

Quadro 9 - Logotipos com cores

\begin{tabular}{|c|c|c|c|c|c|c|c|c|c|c|c|}
\hline Logo \Resposta & \multicolumn{2}{|c|}{ Em branco } & \multicolumn{2}{|c|}{ Não } & $\begin{array}{c}\text { Axial } 2 \\
\text { eixos }\end{array}$ & \multicolumn{2}{|c|}{$\begin{array}{c}\mathrm{X} / \mathrm{sim} \\
\text { Simetria }\end{array}$} & \multicolumn{2}{|c|}{ Rotação } & \multicolumn{2}{|c|}{ eixos } \\
\hline Grupo & G1 & G2 & G1 & G2 & G1 & G1 & $\mathrm{G} 2$ & G1 & G2 & G1 & $\mathrm{G} 2$ \\
\hline & 3 & 3 & 4 & 1 & & 2 & & 3 & 2 & 2 & 2 \\
\hline & 4 & 4 & 2 & & 1 & & 1 & 4 & 3 & & 2 \\
\hline & 5 & 2 & 3 & & & & 1 & 2 & 3 & & 5 \\
\hline
\end{tabular}

Fonte: Os autores. 
Foram consensos: as duas figuras coloridas sugerem uma rotação, mas mesmo que não se considere a cor como um atributo aplicado à figura geométrica, a primeira não permanece invariante após qualquer movimento de rotação, que não seja a identidade, pois a figura geométrica não é simétrica. No entanto, a segunda, sem considerar o atributo da cor, é uma figura simétrica por uma rotação de $180^{\circ}$.

Podemos resumir os principais resultados da análise da atividade diagnóstica:

Os residentes carecem de conhecimento comum do conteúdo sobre as transformações. Alguns reconheceram não lembrar os nomes das isometrias, outros assinalaram simplesmente simetria ou sim (tem simetria) nos casos em que a figura é simétrica, o que pode indicar que também não conheciam as isometrias. Para a maioria dos residentes simetria está associada à existência de um eixo de simetria, visto que mesmo nos logos em que há rotação, precisavam desenhar um eixo de simetria.

A isometria reflexão axial foi reconhecida pelos participantes com mais facilidade quando o eixo é vertical, o que confirma a influência da variável didática da posição do eixo na identificação da reflexão em relação a uma reta. A rotação não faz parte do repertório de conhecimentos da maioria dos residentes, nem do ponto de vista do conhecimento comum, e os participantes responderam rotação sem muita convicção, e sem identificar o ângulo de rotação.

O conhecimento sobre a simetria, apresentado pelos residentes, é mais próximo da forma como aparecem nos livros, superficial e informal, associado quase sempre à reflexão apenas.

\section{Conclusão}

Trouxemos para esse artigo os dados da atividade diagnóstica de uma pesquisa que teve dois questionamentos principais: investigar os conhecimentos sobre as transformações geométricas de professores participantes de uma atividade formativa, e analisar as contribuições de um minicurso sobre as transformações geométricas no sentido de os professores participantes da atividade formativa ampliarem os conhecimentos para o ensino das transformações geométricas, com vistas a que esse conteúdo chegue às salas de aula. As atividades propostas no diagnóstico nos deram indícios de que os participantes possuíam apenas alguns elementos do conhecimento comum/especializado sobre as isometrias. $\mathrm{O}$ diagnóstico nos mostrou que os participantes possuíam apenas alguns elementos do conhecimento comum sobre as isometrias. O conceito de simetria para os residentes está fortemente associado à transformação geométrica reflexão, de forma semelhante ao que é feito nos livros didáticos. Os professores possuem uma percepção da simetria, quando há rotação, mas para justificá-la nesses casos sentem necessidade de incluir um eixo, o que indica um conhecimento limitado no que diz respeito à rotação. É preciso um aprofundamento do conhecimento teórico desses conceitos pelos professores, condição importante para qualquer trabalho com as transformações geométricas em sala de aula.

Visando ampliar os conhecimentos dos professores, trabalhamos, na sequência da pesquisa, buscando favorecer a reflexão sobre formas de apresentação das transformações geométricas que tornem os conceitos mais compreensíveis, incluindo o uso do papel vegetal, dobras e recortes, geoplano, papel quadriculado e recursos tecnológicos como o GeoGebra. Consideramos que as experiências com esses recursos favoreceram não só o conhecimento sobre as estratégias e possibilidades de recursos para o ensino, como também, a vivência, pelos professores, das dificuldades que os alunos podem apresentar na aprendizagem deste conteúdo.

\section{Referências}

Brasil. Ministério da Educação. (2016). PNLD 2017: Matemática - Ensino Fundamental Anos Finais. Brasília: FNDE.

Bulf, C. (2008). Etude des effets de la symetrie axiale sur la conceptualisation des isometries planes et sur la nature du travail geometrique au college. Doutorado em Didactique des Mathématiques, Universite Paris Diderot, Paris.

Grenier, D. (1988). Construction et étude du fonctionnement d'un processus d'enseignement sur la symétrie orthogonale en sixième. Doutorado em Didactique des Mathématiques, Universite Joseph Fourier, Grenoble.

Grenier, D.L. (1987). Tranformations géométriques: le cas de la symétrie orthogonale. didactique et acquisition des conaissances scientifiques. Actes Du Colloque De Sèvres. Sèvres.

Jahn, A. P. (1998). Des transformations des figures aux transformations ponctelles: Étude d'une séquence d'enseignement avec cabri-géomètre. Tese De Doutorado, Universite Joseph Fourier, Grenoble.

Mabuchi, S.T. (2000). Transformações geométricas: A trajetória de um conteúdo ainda não incorporado às práticas escolares nem à formação de professores. Mestrado em Educação Matemática, Pontifícia Universidade Católica, PósGraduação Em Educação Matemática, São Paulo.

Shulman, L.S. (1987). Knowledge and teaching: Foundations of the new reform. Harvard Educational Review.

Shulmann, L.S. (1986). Those who understand: Knowledge Growth In Teaching. Educational Researcher.

Thaqi, X. (2009). Aprender a enseñar transformaciones geométricas en primaria desde una perspectiva cultural Doutorado em Ciências da Educação Matemática, Universidade de Barcelona, Barcelona.

Usiskin, Z. (2014). Transformations in u.s. commercial high school geometry textbooks since 1960: A brief report. Proceedings Of The International Conference On Mathematics Textbook Research And Development,, (Pp. 471-476). Southampton. 